\title{
EQUADIFF 8
}

\section{Alexander V. Ivanov \\ Regularity for doubly nonlinear parabolic equations}

In: Pavol Brunovský and Milan Medved' (eds.): Equadiff 8, Czech - Slovak Conference on Differential Equations and Their Applications. Bratislava, August 24-28, 1993. Mathematical Institute, Slovak Academy of Sciences, Bratislava, 1994. Tatra Mountains Mathematical Publications, 4. pp. 117--124.

Persistent URL: http://dml.cz/dmlcz/700102

\section{Terms of use:}

(C) Comenius University in Bratislava, 1994

Institute of Mathematics of the Academy of Sciences of the Czech Republic provides access to digitized documents strictly for personal use. Each copy of any part of this document must contain these Terms of use.

This paper has been digitized, optimized for electronic delivery and stamped
with digital signature within the project DML-CZ: The Czech Digital Mathematics
Library http://project.dml.cz




\title{
REGULARITY FOR DOUBLY NONLINEAR PARABOLIC EQUATIONS
}

\author{
AleXander V. Ivanov
}

\begin{abstract}
Hölder estimates and existence of regular solutions of CauchyDirichlet problem for doubly nonlinear parabolic equations are established. Similar equations arise in the study of turbulent filtration of a gas or a fluid through porous media.
\end{abstract}

Let $\Omega$ is a bounded open set in $\mathbb{R}^{n}, n \geq 1, Q_{T}=\Omega \times(0, T], S_{T}=$ $\partial \Omega \times(0, T], \Gamma_{T}=S_{T} \times(\Omega \times\{t=0\}) \quad\left(\Gamma_{T}\right.$ is a parabolic boundary of $\left.Q_{T}\right)$. Consider in $Q_{T}$ equation of the type

$$
\partial u / \partial t-\operatorname{div} \vec{a}(x, t, u, \nabla u)+a_{0}(x, t, u, \nabla u)=0,
$$

where $\vec{a}=\left(a^{1}, \ldots, a^{n}\right), \nabla u=\left(\frac{\partial u}{\partial x_{1}}, \ldots, \frac{\partial u}{\partial x_{n}}\right)$. Assume that $a^{i}(x, t, u, p)$, $a_{0}(x, t, u, p)$ satisfy the Caratheodory condition and let for a.e. $(x, t) \in Q_{T}$ and any $u \in \mathbb{R} \backslash\{0\}, p \in \mathbb{R}^{n}$ inequalities

$$
\begin{aligned}
& \vec{a}(x, t, u, p) \cdot p \geq \nu_{0}|u|^{l}|p|^{m}-\Phi_{0}(x, t, u), \quad \nu_{0}>0 ; \\
& |\vec{a}(x, t, u, p)| \leq \mu_{1}|u|^{l}|p|^{m-1}+\Phi_{1}(x, t, u),
\end{aligned}
$$

hold with some $m>1, l>1-m$ and $\Phi_{i}(x, t, u) \geq 0$.

The prototypes of equations of the type (1.1), (1.2) are

$$
\partial u / \partial t-\operatorname{div}\left\{|u|^{l}|\nabla u|^{m-2} \nabla u\right\}=0, \quad m>1, l>1-m
$$

and

$$
\partial u / \partial t-\sum_{i=1}^{n} \frac{d}{d x_{i}}\left\{|u|^{l}\left|\partial u / \partial x_{i}\right|^{m-2} \partial u / \partial x_{i}\right\}=0, \quad m>1, l>1-m .
$$

Equations (1.3), $\left(1.3^{\prime}\right)$ and close equations arise in the study of turbulent filtration of a gas or a liquid through porous media and non-Newtonian fluids.

AMS Subject Classification (1991): $35 \mathrm{~K} 55$, 35K65.

Key words: regularity, turbulent flow, porus media. 
For example in the case $m>1, l>0$ equation (1.3) is called in the survey [1] as the equation of non-Newtonian polythropic filtration. We shall use this title for (1.3) further only in the case $m>2, l>0$.

On the other hand in the case $n=1, m \in[3 / 2,2), l \geq m-1$ equation (1.3) arises in the study of turbulent flow of a gas in one-dimensional porous media. This phenomenon was described at first by Leibenson in [2]. Further we shall call (1.3) as the Leibenson equation for any $n \geq 1, m \in(1,2), l>0$, $m+l \geq 2$.

At last in the study of flow through three-dimensional porous media in turbulent regimes (i.e., in the case when in view of large velocities the classical Darcy's law fails to be true) several authors (see [3]) consider equation of the type

$$
\partial u / \partial t-\operatorname{div}\left\{\left.\left.|u|^{l}\left|\nabla u-c_{0}\right| u\right|^{k} \vec{Z}\right|^{m-2}\left(\nabla u-c_{0}|u|^{k} \vec{Z}\right)\right\}=0
$$

with $m \in(1,2), l=(s-1)(m-1), k=r-s+1, \vec{Z}=(0,0,1)$, from the physical point of view it is natural to assume that $1<s \leq r$. Thus we have in (1.4) $l>0, k \geq 1$. Furthermore we shall call on equation (1.4) briefly as the equation of turbulent filtration.

In the case of equation (1.3) we have

$$
a^{i}=|u|^{l}|p|^{m-2} p_{i}, \quad \frac{\partial a^{i}}{\partial p_{j}} \xi_{i} \xi_{j} \geq \min (1, m-1)|u|^{l}|p|^{m-2}|\xi|^{2} .
$$

In view of (1.5) it is natural to use the following classification for equations (1.1), (1.2):

$$
\begin{array}{llll}
m>2, l>0 & 1<m<2, l>0 & m>2, l<0 & 1<m<2, l<0 \\
\text { doubly degene- } & \text { singular-degene- } & \text { degenerate- } & \text { doubly singular } \\
\text { rate par. eq. } & \text { rate par. eq. } & \text { singular par. eq. } & \text { par. eq. }
\end{array}
$$

On the other hand rewrite $\left(1.3^{\prime}\right)$ as

$$
\partial u / \partial t-D(u, \nabla u) \Delta u+\mathcal{E}(u, \nabla u)=0,
$$

where $D(u, p)=(m-1)|u|^{l} \sum_{i=1}^{n}\left|p_{i}\right|^{m-2}, \quad \mathcal{E}(u, p)=l|u|^{l-2} u \sum_{i=1}^{n}\left|p_{i}\right|^{m}$. It is evident that

$$
\lim _{\mu \downarrow 0} D(\mu u, \mu p)= \begin{cases}0, & \text { if } m+l>2, \\ D(u, p), & \text { if } m+l=2, \\ +\infty, & \text { if } m+l>2 .\end{cases}
$$

In view of (1.6) we shall use also the following classification for general equations (1.1), (1.2):

$$
m+l>2
$$

equations of the type of slow diffusion

$$
m+l=2
$$

equations of the type of normal diffusion

$$
m+l<2
$$

equations of the type

of fast diffusion 
So the equation of non-Newtonian polythropic filtration is a doubly degenerate parabolic equation and at the same time is an equation of the type of slow diffusion, while the Leibenson equation is a singular-degenerate parabolic equation and an equation of the type of slow or normal diffusion. On the other hand the equation of turbulent filtration is a singular-degenerate parabolic equation and an equation of the type of fast diffusion if $s<\frac{1}{m-1}$, of normal diffusion if $s=\frac{1}{m-1}$ and of slow diffusion if $s>\frac{1}{m-1}$.

Remark now that equations (1.1), (1.2) can be rewritten as equations of the type

$$
\frac{\partial b(v)}{\partial t}-\operatorname{div} \vec{A}(x, t, v, \nabla v)+A_{0}(x, t, v, \nabla v)=0
$$

where

$$
v=|u|^{\sigma} u, \quad \sigma=\frac{l}{m-1}, \quad b(v)=|v|^{\beta-1} v, \quad \beta=\frac{1}{\sigma+1}=\frac{m-1}{l+m-1}
$$

and coefficients $A^{i}(x, t, v, q)$ satisfy the following inequalities

$$
\begin{aligned}
\vec{A}(x, t, v, q) \cdot q & \geq \hat{\nu}_{0}|q|^{m}-\hat{\Phi}_{0}(x, t, v), \quad \hat{\nu}_{0}>0 \\
|\vec{A}(x, t, v, q)| & \leq \hat{\mu}_{1}|q|^{m-1}+\hat{\Phi}_{1}(x, t, v),
\end{aligned}
$$

with $m>1, \beta>0, \hat{\Phi}_{i}(x, t, v) \geq 0$. It is evident that equations of the type (1.7), (1.8) are only another form of equations of the type (1.1), (1.2).

Equations (1.7), (1.8) (and hence (1.1), (1.2)) are known as doubly nonlinear parabolic equations. Existence results for equations of this type were obtained in the pioneering papers $[4,5]$ by $\mathrm{R}$ aviart and J.-L. $\mathrm{L}$ i o $\mathrm{n}$ s and then in $[6-13,3]$ etc. The purpose of this talk is to discuss the regularity problem for equations (1.1), (1.2). Up to recent time there were no regularity results for weak solutions of doubly nonlinear parabolic equations. Under regular solution we mean in this paper Hölder continuous weak solution. The simple modification of B a r e $\mathrm{n} \mathrm{b} \mathrm{l} \mathrm{a} \mathrm{t} \mathrm{t}$ explicit self-similar solution (see [14]) lets to show that holderness is the best possible smoothness of weak solutions (1.1), (1.2) in the case $m>1, l>1$.

More precisely this paper devotes mainly to discussion of two problems for equations (1.1), (1.2):

1. regularity (Hölder continuity) of every bounded weak solution;

2. existence of regular solution of Cauchy-Dirichlet problem.

For discussion of the first of these problems it is necessary to specify the growth conditions (1.2) for equation (1.1). Suppose that for a.e. $(x, t)$ and any $u \in \mathbb{R}, p \in \mathbb{R}^{n}$

$$
\begin{aligned}
\vec{a}(x, t, u, p) \cdot p & \geq \nu_{0}|u|^{l}|p|^{m}-\varphi_{0}, & & \nu_{0}>0 \\
|\vec{a}(x, t, u, p)| & \leq \mu_{1}|u|^{l}|p|^{m-1}+\varphi_{1}|u|^{\alpha}, & & \alpha=l / m ; \\
\left|a_{0}(x, t, u, p)\right| & \leq \mu_{2}|u|^{l m^{\prime}}|p|^{m}+\varphi_{2}, & & 1 / m+1 / m^{\prime}=1,
\end{aligned}
$$


where $m>1, l>1-m, \varphi_{i}=\varphi_{i}(x, t) \geq 0, \varphi_{0} \equiv \varphi_{1} \equiv 0$ if $l<0 ; \varphi_{0}, \varphi_{1}^{m^{\prime}}$, $\varphi_{2} \in L_{q, q_{0}}\left(Q_{T}\right)$ with appropriate exponents $q, q_{0}$. For the sake of simplicity we shall assume however that $\varphi_{0}, \varphi_{1}$ and $\varphi_{2}$ are some given constants.

Recall that the pioneering results and methods of establishing of Hölder estimates for weak solutions of linear and quasilinear uniformly elliptic and parabolic equations in divergence form appeared in the classical papers by D e G i o r g i, $\mathrm{N}$ as h, Moser and Ladyzhenskaya-Uraltseva.

Hölder continuity of weak solutions of quasilinear uniformly parabolic equations of the type (1.1), (1.9) (i.e., in the case $m=2, l=0$ ) were established with the aid of different methods in sixtieth by Ladyzhenskay aUraltseva, Aronson-Serrin, author and others (see in particular [15-19]).

Continuity or Hölder continuity of weak solutions of different subclasses of (1.1), (1.9) in the case $m=2, l \neq 0$ were proved only in eightieth in [20-29].

In 1986 the important advancement was made by $\mathrm{Di}$ B e nedet to who was able to establish Hölder estimates for (1.1), (1.9) in the case $m>2, l=0$ ([30]). In particular an interesting development of approaches by $\mathrm{D} \mathrm{e} \mathrm{G}$ i o r g i and $\mathrm{L}$ adyzhenskaya-Uraltse va of establishing of Hölder estimates is given in [30]. Later in [31] Hölder estimates in the singular case $1<m<2$, $l=0$ were obtained under additional assumptions concerning the structure of equation and properties of weak solution.

Hölder estimates for multidimensional $(n>1)$ weak solutions of quasilinear doubly degenerate parabolic equations (i.e., for (1.1), (1.9) in the case $m>2$, $l>0$ ) were obtained in 1989 by myself with the aid of appropriate development of $\mathrm{D}$ i $\mathrm{B}$ e n e d e t t o approaches (see [32-34, 37, 38]). These estimates were used in $[35,36]$ for proving of existence of nonnegative Hölder continuous weak solutions of Cauchy-Dirichlet problem for (1.1), (1.9) in the case $m>2, l>0$. In all my papers I considered only nonnegative weak solutions namely because they have physical sense. More precisely I established Hölder estimates for the following (natural) class of weak solutions.

DEFINITION 1.1. Any nonnegative bounded in $Q_{T}$ function $u$ is a weak solution [supersolution, subsolution] if

a) $u \in C\left([0, T] ; L_{1}(\Omega)\right), \quad \partial u^{\sigma+1} / \partial x_{i} \in L_{m}\left(Q_{T}\right), \quad \sigma=\frac{l}{m-1}, \quad i=1, \ldots, n$;

b) for any $\Phi \in \stackrel{\circ}{C}^{1}\left(Q_{T}\right)$ and any $t_{1}, t_{2} \in[0, T]$

$$
\begin{gathered}
\left.\int_{\Omega} u \Phi d x\right|_{t_{1}} ^{t_{2}}+\int_{t_{1}}^{t_{2}} \int_{\Omega}\left\{-u \Phi_{t}+\vec{a}\left(x, t, u, u_{x}\right) \cdot \nabla \Phi+a_{0}\left(x, t, u, u_{x}\right) \Phi\right\} d x d t=0 \\
{[\geq 0, \leq 0]}
\end{gathered}
$$


where $u_{x}=\left(u_{x_{1}}, \ldots, u_{x_{n}}\right)$ and $u_{x_{i}}$ are defined by

$$
u_{x_{i}}=\left\{\begin{array}{ll}
(1+\sigma)^{-1} u^{-\sigma} \partial \hat{u} / \partial x_{i} & \text { in }\left\{Q_{T}: u>0\right\} \\
0 & \text { in }\left\{Q_{T}: u=0\right\}
\end{array}, \hat{u}=u^{\sigma+1} \in W_{m}^{1.0}\left(Q_{T}\right) .\right.
$$

Remark that Definition 1.1 is available in the general case $m>1, l>1-m$. It should be to mention that in the case $n=1, m>1, l>2-m$ Hölder continuity in $\mathbb{R}^{1} \times[\varepsilon, T]$ for any $\varepsilon>0$ with the best possible Hölder exponent

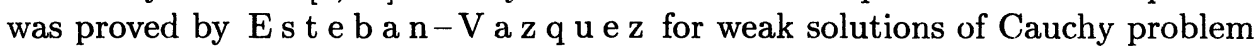
(see [39]).

Recently some new investigations of regularity for doubly nonlinear parabolic equations appeared. In preprint [40] in the case $m>2, l>0$ Hölder estimates for weak solution belonging to $W_{m}^{1,0}\left(Q_{T}\right)$ are obtained without assumption that weak solution is nonnegative. On the other hand using approaches of paper [31] $\mathrm{V}$ e s p ri in [42] established Hölder estimates in the singular case $1<m<2$, $l \neq 0$ under (roughly speaking) the following additional assumptions:

(i) $\vec{a}=\vec{a}(x, u, p), \quad[\vec{a}(x, u, p)-\vec{a}(x, u, q)] \cdot(p-q) \geq 0$;

(j) functions $u \rightarrow a^{i}(x, u, p)$ are Lipschitz $(i=1, \ldots, n)$;

(k) $u \in W_{m}^{1,0}\left(Q_{T}\right)$;

(l) $\partial u / \partial t \in L_{2}\left(Q_{T}\right)$.

It should be to say that Hölder estimates established in [31, 42] are independent of Lipshitz constants in (j) and norms $\|\nabla u\|_{L_{m}\left(Q_{T}\right)},\|\partial u / \partial t\|_{L_{2}\left(Q_{T}\right)}$. Moreover authors of $[31,42]$ suppose that weak solution under consideration is a weak $W_{m}^{1,0}\left(Q_{T}\right)$ - limit of solutions $u_{\varepsilon}$ of some regularized equations for which conditions (1.9) and $(i)-(l)$ are fulfilled. But in any case condition (l) is non-pleasant with the point of view of proving of existence of regular solution of Cauchy-Dirichlet problem (because it is difficult to find a regularized problems for which all conditions (1.2) and $(i)-(l)$ would be fulfilled). As far as we know up to present nobody has proved the existence of a Hölder continuous weak solution of the Cauchy-Dirichlet problem in the singular case $1<m<2$.

Recently I established some new results concerning Hölderness and existence of regular solutions for doubly nonlinear parabolic equations of the type (1.1), (1.2) which I consider here either in the case

$$
\text { (SN) } \quad m>1, \quad l \geq 0, \quad m+l \geq 2
$$

or in the case

$$
\text { (F) } \quad m>1, \quad 1<m+l<2 \text {. }
$$

So in the case (SN) I deal with equations of the type of slow or normal diffusion under additional assumption $l \geq 0$, while in the case (F) I consider the full class of equations of the type of fast diffusion. 
At first I establish Hölder estimates. If in the former case the establishment of these estimates requires only the structure conditions (1.9), in the latter case I have to assume beside (1.9) additional conditions (i), (j), (k) (but not (l)). Thus in particular as against of $\mathrm{V}$ e s p ri result [41] I eliminate in the singular case $1<m<2$ all additional conditions if $m+l \geq 2, l \geq 0$ and condition (l) if $m+l<2$. These results will be published in [43-45].

We applied these Hölder estimates for proving of existence of Hölder continuous solutions of Cauchy-Dirichlet problem for equations of the type (1.1), (1.2) with parameters satisfying conditions

$$
m>\max (2 n /(n+2), 1), \quad l \geq 0 .
$$

Moreover we proved that regular solution of Cauchy-Dirichlet problem is unique. In particular existence and uniqueness of regular solution of Cauchy-Dirichlet problem is established for nonhomogeneous equations with the principal parts like in (1.3) and (1.4) (see [43], [46]).

\section{REFERENCES}

[1] KALASHNICOV, A. S.: Some problems of the qualitative theory of nonlinear degenerate second order parabolic equations, Russian Math. Surveys 42 (1987), 169-222.

[2] LEIBENSON, L. S.: General problem of the movement of a compressible fluid in a porous medium, Izv. Akad. Nauk, SSSR, Geography and Geophysics 9 (1945), 7-10.

[3] DIAZ, J. I.-de TELLIN, F.: On doubly nonlinear parabolic equation arising in some models related to turbulent regimes, preprint (1991), 1-50.

[4] RAVIART, P. A.: Sur la resolution de certaines equations paraboliques non lineaires, J.Funct. Anal. 5 (1970), 299-328.

[5] LIONS, J. L.: Quelques Methodes de Resolution de Problemes aux Limites non Lineaires, Dunod, Paris, 1969.

[6] BAMBERGER, A.: Etude d'une equation doublement non lineaire, J. Funct. Anal. 24 (1977), 148-155.

[7] GRANGE, O.-MIGNOT, F.: Sur la resolution d'une equation et d'une inequation paraboliques non lineairs, J. Funct. Anal 11 (1972), 77-92.

[8] ARAI, T.: On the existence of solutions for doubly nonlinear parabolic equations, Res. Repts. Inst. Inform. Sci. Technol. 6 (1980), 45-57.

[9] KALASHNIKOV, A. S.: The Cauchy problem for degenerate second-order parabolic equations with non-power non-lineairities, Trudy Sem. Petrovsk. 6 (1981), 83-96.

[10] ALT, H. W.-LUCKHAUS, S.: Quasi-linear elliptic-parabolic differential equations, Math. Z. 183 (1983), 311-341.

[11] TSUTSUMI, M.: On solutions of some doubly nonlinear degenerate parabolic equations with absorption, J. Math. Anal. Appl. 132 (1988), 187-212.

[12] BLANCHARD, D.-FRANCFORT, G.: Study of a doubly nonlinear heat equation with no growth assumption on the parabolic term, SIAM J. Math. Anal. 19 (1988), 1032-1056.

[13] JINGXUE, Y.: On a class of quasilinear parabolic equations of second order with double degeneracy, J. Partial Differentiall Equations 3 (1990), 49-64. 


\section{REGULARITY FOR DOUBLY NONLINEAR PARABOLIC EQUATIONS}

[14] BARENBLATT, G. I.: On some unsteady motions of a fluid and a gas in a porous medium, Prik. Mat. Mekh. 16 (1952), 67-78.

[15] LADYZHENSKAYA, O. A.-SOLONNIKOV, V. A.-URALTSEVA, N. N.: Linear and Quasilinear Equations of Parabolic Type, Nauka, Moscow, 1967; English transl. in Amer. Math. Soc., Providence, R. I. (1968).

[16] OLEINIK, O. A.-KRUZHKOV, S. N.: Qusilinear second order parabolic equations with several independent variables, Uspekhi Mat. Nauk 16 5(101) (1961), 115-155.

[17] ARONSON, J. G.-SERRIN, J. : Local behavior of solutions of quasilinear parabolic equations, Arch. Rational Mech. Anal. 25 (1967), 81-122.

[18] IVANOV, A. V.: Harnack's inequality for generalized solutions parabolic equations of second order, Trudy Mat. Inst. Steklov 102 (1967), 51-84.

[19] TRUDINGER, N. S.: Pointwise estimates and quasi-linear parabolic equations, Comm. Pure Appl. Math. 21 (1968), 205-226.

[20] CAFFARELLI, L. A.-FRIEDMAN, A.: Regularity of the free-boundary of a gas in a n-dimensional porous medium, Indiana Univ. Math. J. 29 (1980), 361-369.

[21] CAFFARELLI, L. A.-EVANS, L. C.: Continuity of the temperature in the two phase Stefan problem, Arch. Rational Mech. Anal. 81 (1983), 199-220.

[22] SACKS, P. E.: Continuity of solutions of a singular parabolic equation, Nonlinear Anal., TMA 7 (1983), 387-409.

[23] Di BENEDETTO, E.: Continuity of weak solutions to a general porous medium equation, Indiana Univ. Math. J. 32 (1983), 83-118.

[24] ZIEMER, W.: Interior and boundary continuity of weak solutions of degenerate parabolic equations, Trans. Amer. Math. Soc. 271 (1982), 733-748.

[25] IVANOV, A. V.: On estimate of modulus of continuity of weak solutions of some singular parabolic equations, Zap. Nauchn. Sem. Leningrad. Otdel. Mat. Inst. Steklov. (LOMI) 147 (1985), 49-72.

[26] YA-ZHE, CHEN : Hölder estimates for solutions of uniformly degenerate parabolic equations, Chinese Ann. Math. 5b (1984), 666-678.

[27] Di BENEDETTO, E.-FRIEDMAN, A.: Hölder estimates for nonlinear degenerate parabolic systems, J. Reine Angew. Math. 357 (1985), 83-127.

[28] IVANOV, A. V.: Estimates of the Hölder constant for weak solutions of degenerate parabolic equations, Zap. Nauchn. Sem. Leningrad. Otdel. Mat. Inst. Steklov. (LOMI) 152 (1986), 21-44.

[29] WIEGNER, M.: On $C_{\alpha}$-regularity of the gradient of solutions of degenerate parabolic system, Universität Bonn, preprint 682 (1984).

[30] Di BENEDETTO, E. : On the local behaviour of solutions of degenerate parabolic equations with measurable coefficients, Ann. Sc. Norm. Sur. 13 (1986), 485-535.

[31] YA-ZHE, CHEN-Di BENEDETTO, E.: On the local behaviour of solutions of singular parabolic equations, Arch. Rational Mech. Anal. 103 (1983), 319-346.

[32] IVANOV, A. V.: Hölder estimates for weak solutions of quasilinear doubly degenerate parabolic equations, Zap. Nauchn. Sem. Leningrad. Otdel. Mat. Inst. Steklovi. (LOMI) 171 (1989), 70-105.

[33] IVANOV, A. V.: Uniform Hölder estimates for weak solutions of quasilinear doubly degenerate parabolic equations, Algebra i Analiz 32 (1991), 139-179; English transl. in St. Petersburg Math. J. 3 (1992), 363-403.

[34] IVANOV, A. V.: Boundary Hölder estimates for weak solutions of quasilinear doubly degenerate parabolic equations, Zap. Nauchn. Sem. Leningrad. Otdel. Mat. Inst. Steklov. (LOMI) 183 (1991), 45-69. 
[35] IVANOV, A. V.-MKRTCHAN, P. Z.: On the existence Hölder continuous weak solutions of the first boundary value problem for quasilinear doubly degenerate parabolic equations, Zap. Nauchn. Sem. Leningrad. Otdel. Mat. Inst. Steklov. (LOMI) 182 (1990), 5-28.

[36] IVANOV, A. V.-MKRTCHAN, P. Z.: On the regularity up to the boundary of weak solutions of Cauchy-Dirichlet problem for quasilinear doubly degenerate parabolic equations, Zap. Nauchn. Sem. Leningrad. Otdel. Mat. Inst. Steklov. (LOMI) 191 (1991), 83-98.

[37] IVANOV, A. V.: The classes $B_{m, l}$ and Hölder estimates of weak solutions for quasilinear doubly degenerate parabolic equations, POMI preprints, 1991, E-11-91, 3-66; E-12-91, 3-51.

[38] IVANOV, A. V.: The classes $B_{m, l}$ and Hölder estimates of weak solutions for quasilinear parabolic equations admitting doubly degeneracy, Zap. Nauchn. S. Peterburg. Otdel. Mat. Inst. Steklov. (POMI) 197 (1992), 42-70.

[39] ESTEBAN, J. R.-VAZQUES, J. L.: On the equation of turbulent filtration in one-dimensional porous media, Nonlinear Anal., TMA 10 (1989), 1303-1325.

[40] PORZIO, M. M.-VESPRI, V.: Hölder estimates for local solutions on some doubly nonlinear parabolic equations, preprint (1992), 1-47.

[41] VESPRI, V.: On the local behaviour of solutions of a certain class of doubly nonlinear parabolic equations, Manuscripta Math. 75 (1992), 65-80.

[42] VESPRI, V.: Harnack type inequalities for solutions of certain doubly nonlinear parabolic equations, Universita degli studi di Milano 32 (1991), 1-23.

[43] IVANOV, A. V.: Quasilinear doubly degenerate and singular-degenerate equations, to appear in "Vortragsreihe 1992", Mathematishes Institut der Bonn Universität.

[44] IVANOV, A. V.: Equations of the type of slow and normal diffusion, to appear in Zap. Nauchn. Sem. Leningrad. Otdel. Mat. Inst. Steklov. (POMI).

[45] IVANOV, A. V.: Equations of the type of fast diffusion, to appear in St. Petersburg Math. J.

[46] IVANOV, A. V.-JAEGER; W.: Existence and uniqueness of a regular solution of Cauchy-Dirichlet problem for the three-dimensional equation of turbulent filtration (to appear).

[47] GILDING, B. J.-PELETIER, L. A.: The Cauchy Problem for an equation in the theory of infiltration, Arch. Rational Mech. Anal. 61 (1976), 127-140.

Received November 29, 1993

Russian Academy of Sciences
Steklov Mathematical Institute
Fontanka 27
191011 Sankt Peterburg
RUSSIA

E-mail: avivanov@lomi.spb.su 